\title{
Oligometastatic Recurrent Ovarian Cancer Treated Using Stereotactic Body Radiotherapy
}

\author{
(D) Satya NARAYAN, ${ }^{1}$ (D) Puneet PAREEK, ${ }^{1}$ (D) Tej Prakash SONI, ${ }^{(1)}$ Sweta SONI, ${ }^{1}$ Puneet BAGRI ${ }^{1}$ \\ 'Department of Radiation Oncology, AlIMS, Jodhpur-Hindistan \\ ${ }^{2}$ Department of Radiation Oncology, Bhagwan Mahaveer Cancer Hospital \& Research Centre, Jaipur-Hindistan
}

\begin{abstract}
SUMMARY
Ovarian cancer is one of the most aggressive and frequent gynecological cancers. Despite an aggressive approach; patients often recur. The treatment options at recurrence are limited, although additional systemic therapy is usually administered. In the recent era, stereotactic body radiation therapy (SBRT) to the area(s) of the oligo-metastatic re-current disease can provide adequate tumor response with good local control. Here, we report a case of 48 years old female, diagnosed with recurrent metastatic ovarian malignancy, successfully treated with the use of SBRT. SBRT is well tolerated with low toxicity rates and effective means of oligo-metastasis control.
\end{abstract}

Keywords: Ovarian cancer; oligo-metastasis; stereotactic body radiotherapy.

Copyright $\odot$ 2020, Turkish Society for Radiation Oncology

\section{Introduction}

Oligo-metastatic ovarian cancer is a state of limited metastatic disease $\leq 3$ sites that may be amenable to aggressive local therapy to gain good local control and disease-free survival. In recent radiation technology era, SBRT has be-come a viable treatment option for selected cases with oligo-metastatic disease. Ovarian cancer remains one of the most aggressive gynecological cancers, and the most frequent cause of death among them.[1] Patients with ovarian cancer frequently develop metastatic disease even after current standard of care treatment.

\section{Case Report}

Forty eight years old premenopausal lady with history of ovarian malignancy in 2015 , treated using neo-adjuvant chemotherapy followed by debulking procedure (exploratory laprotomy with hysterectomy with bilateral salpingo-oophorectomy with infracolic omentec- tomy with pelvic lymphadenectomy) followed by adjuvant chemotherapy. Later, she was on regular follow up every three monthly with CA 125 and imaging-based monitoring. Approximate-ly 14 months from the last chemotherapy, she was presented with complaints of pain abdomen and decreased appe-tite. On PET, CT (Fig. 1b) scan showed hypodense area in segment II $(3.1 \times 3.1 \mathrm{~cm})$ and segment $\mathrm{V}(2.2 \times 2.1 \mathrm{~cm})$ of the liver with the L1 vertebral lesion (SUV 8.27) and left-sided pelvic nodule $(2 \times 2 \mathrm{~cm})$. The patient was planned for radiation treatment. The optimum treatment plan was generated using Rapid Arc based SBRT and doses to liver Segment II 30 Gy in six fractions. In view of $>1 \mathrm{~cm}$ motion target with respiration cycle and very close proximity $(0.5 \mathrm{~cm})$ to Stomach and $40 \mathrm{~Gy}$ in five fractions in one week to L1 vertebra, SOL Liver Segment V, Left common iliac node and left pelvic nodule. Image verification was done daily with $\mathrm{CBCT}$ scans.

After one month of EBRT completion, she underwent two cycles of palliative chemotherapy (Nab-Pa- 


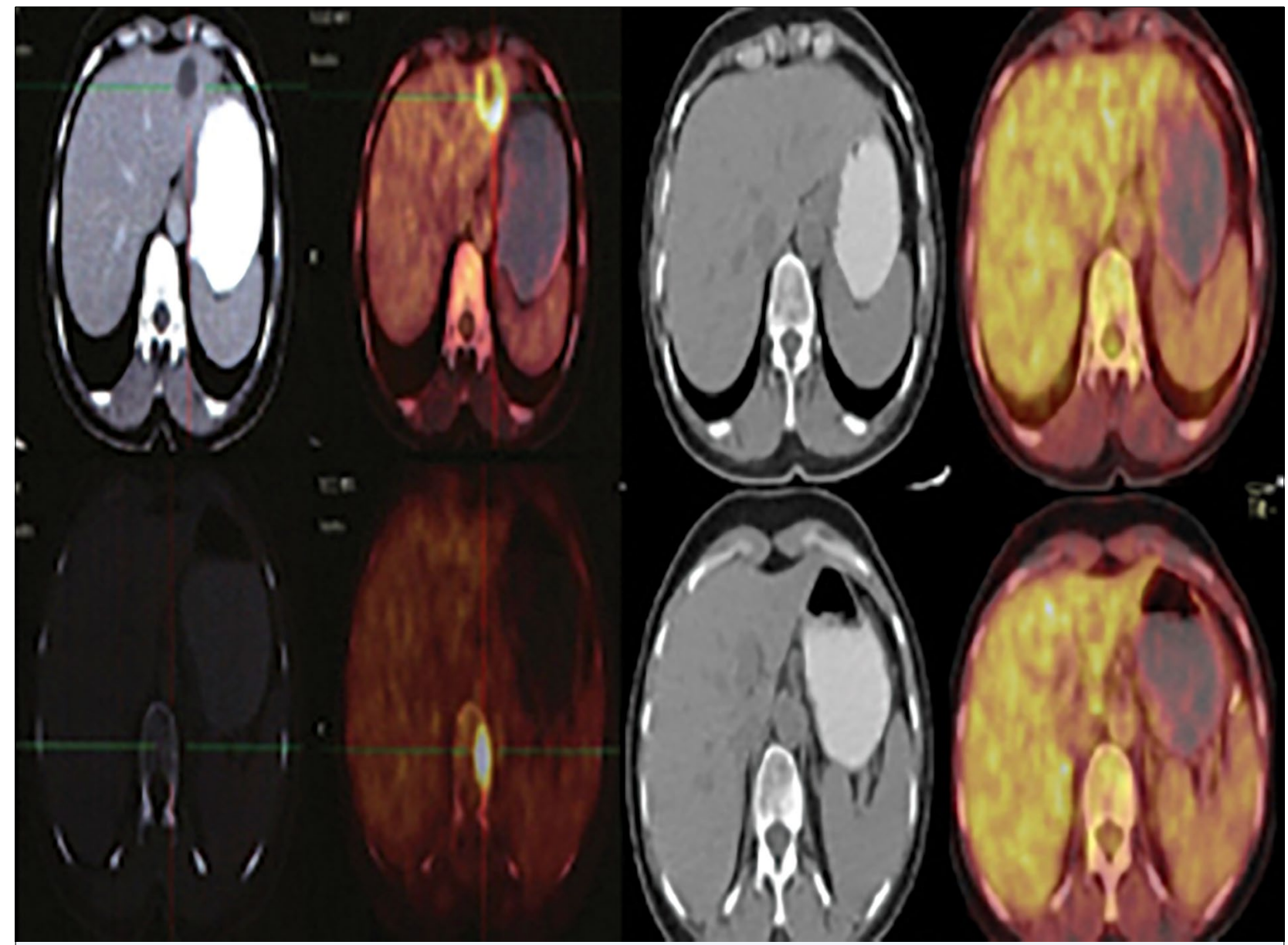

Fig. 1. (a) CECT image and (b) PET-CT image at the time of recurrence (c) PET-CT image after SBRT treatment and after 24 months of treatment.

clitaxel and Carboplatin). Tumor response was evaluated three months of the last cycle of Chemotherapy by PET-CT, according to Response Evaluation Criteria in Solid Tumors (RECIST). The treatment was very well tolerated by the patient and showed a major radiological response. The toxicity and tumor response was scored using the Radiation Therapy Oncology Group (RTOG)/European Organization for Research and Treatment of Cancer (EORTC) Scale. Follow-up is continuing and two years of post-chemotherapy. She still has no signs of relapse, neither on PET-CT scans (Fig. 1c) nor biochemically (CA 125 Values, Fig. 2), and she remains in excellent clinical condition. Consent was obtained for this case report.

\section{Discussion}

The recent advancement in radiation therapy has enabled the delivery of highly conformal, ablative doses of RT to multiple extracranial sites, known as stereotactic body radiotherapy (SBRT). This recent treatment delivery modality improved the biological effectiveness of radiation treatment, which means allowing the reduction of healthy tissue irradiation and increasing the total tumor dose. SBRT technology is also known as stereotactic ablative radiotherapy (SABR). In comparison to conventionally fractionated radiotherapy, which mostly involves daily doses of 1.8 to 2.0 Gy delivered over six to eight weeks, SBRT bring into effect higher doses per treatment (6-30 Gy) delivered over a shorter time frame (typically 1-5 fractions over 1-2 weeks). [2] SBRT is a linear accelerator-based focal radio-therapy delivered with the rigid patient and tumor immobilization, elegant dosimetry, and daily image guidance for verification of setting up. SBRT implies a high-dose per fraction and is delivered in $2-5$ fractions. SBRT serves to decrease tumor burden, destroy chemo-resistant tumor clones, and help stimulate an innate immune response or ex-pose tumor neo-antigens, providing excellent 


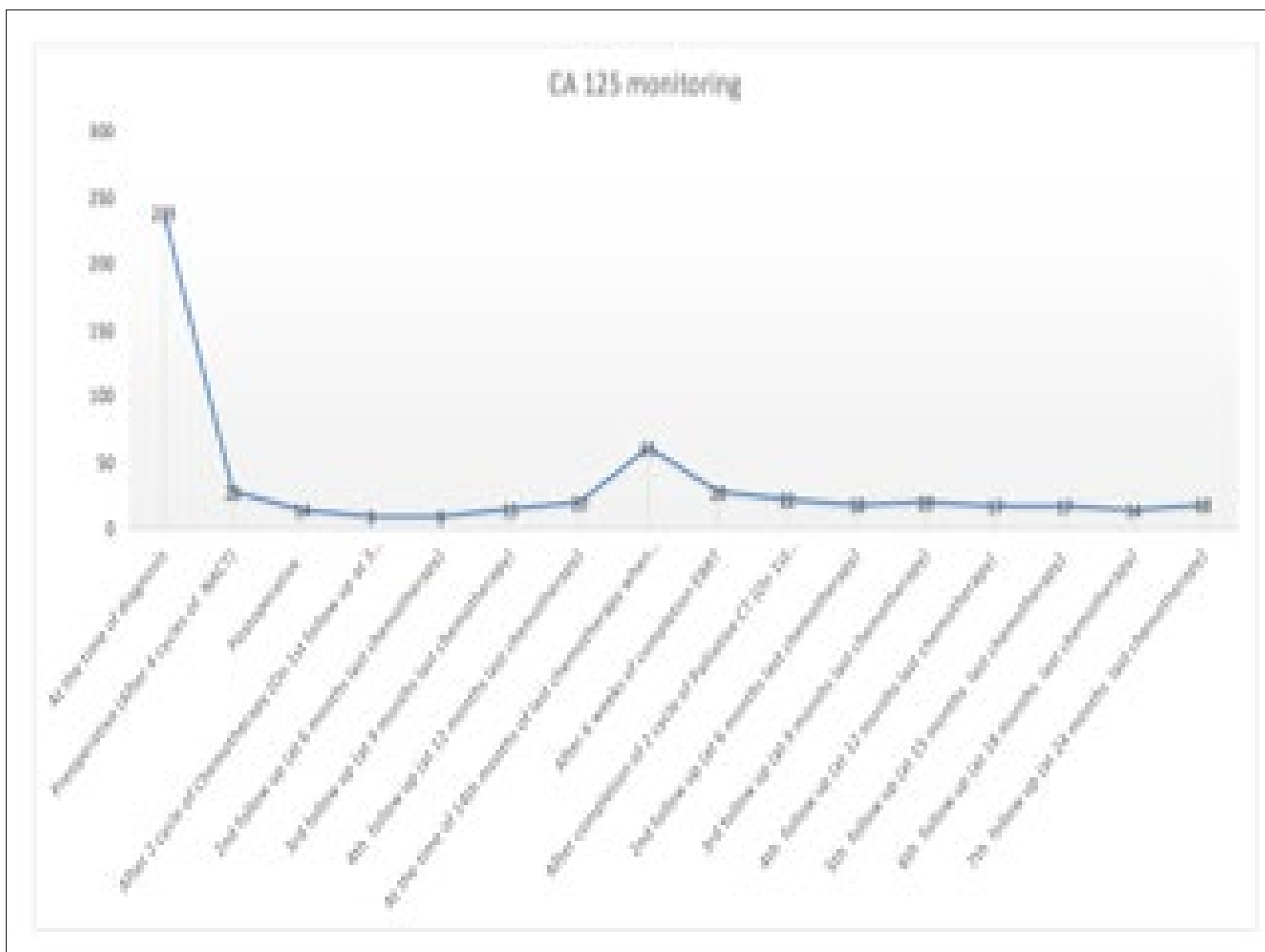

Fig. 2. CA 125 Monitoring pre and post therapy.

rates of local control, minimal acute and late toxicities, and can be used in women who have had prior radiotherapy.

Prognosis of metastatic ovarian cancer is poor, but it is a radiosensitive disease. The use of radiation treatment in ovarian malignancy was drastically compromised due to toxicity issues. The toxicity was basically because of whole abdomino-pelvic (WAP) radiotherapy using the "moving strip" technique.[3] The comprised radiation use has partially been resolved by the development of modern techniques, i.e., stereotactic body radiotherapy (SBRT). Considering the high local control rates for oligo-metastases $(70-90 \%$ at two years) and a low toxicity profile (up to less than $10 \%$ grade three toxicity) have been reported for SBRT in several types of malignancy.[4] Table 1 summarizes few studies of SBRT in the management of metastatic gynecologic malignancies have been published in the literature, including metastatic ovarian cancer.[5-7] In a Phase II study, Cleveland SBRT trial Kunos et al. treated 50 females $(50 \%$ with primary ovarian cancer) with $\leq 4$ sites of metastatic disease with SBRT to a dose 8 Gy $\times 3$ fractions us-ing Cyber-knife. Vallow L et al. suggest that up to $5 \mathrm{~cm}$. size lesion was treated by SBRT at Mayo Clinic Florida per protocol in Liver Oligometastasis in Phase I study. Common treatment sites included para-aortic nodes (38\%), pelvic nodes $(28 \%)$, and the liver (16\%). The treatment outcome of median disease-free survival was 7.8 months and overall survival was 20.2 months, with only 3 grade $\geq 3$ toxicities. The local control rate for abdominal lymph nodes and hepatic metastasis suggest promising results in various published studies. Here, we present a case of re-current metastatic ovarian cancer that remains disease-free with excellent performance status and less toxicity at two years after receiving SBRT.

SBRT serves to decrease tumor burden, help stimulate innate immune response or expose tumor neo antigens and, destroy chemo-resistant tumor clones, providing excellent rates of local control with minimal 


\begin{tabular}{|c|c|c|c|c|}
\hline Study & Year & Major Site(s) of lesion(s) & $\begin{array}{l}\text { Number of } \\
\text { lesions }\end{array}$ & Doses prescribed \\
\hline University of California Mesko et al.[7] & 2017 & $\begin{array}{l}\text { Recurrent/metastatic } \\
\text { ovarian, vaginal, cervical, } \\
\text { endometrial cancers }\end{array}$ & $\geq 1$ & $\begin{array}{c}\text { Median of } \\
8 \mathrm{~Gy} \times 5 \text { fractions }\end{array}$ \\
\hline J.M. Rohann et al.[6] & 2016 & $\begin{array}{l}\text { Abdomino-pelvic LN, } \\
\text { Liver, Lung, Para vaginal mass }\end{array}$ & A & $\begin{array}{l}\text { Abdomino-pelvic LN 36-45Gy/6fr, } \\
\text { Liver } 67.5-75 \mathrm{~Gy} / 3 \mathrm{fr}, \\
\text { Lung } 48 \mathrm{~Gy} / 4 \mathrm{fr}, \\
\text { Para-vaginal mass } 36 \mathrm{~Gy} / 6 \mathrm{fr}\end{array}$ \\
\hline Ohio Kunos et al.[5] & 2012 & $\begin{array}{l}\text { Metastatic sites, ovarian, } \\
\text { primary peritoneal, } \\
\text { endometrial cancers }\end{array}$ & $\leq 4$ & $\begin{array}{l}\text { Carboplatin+Gemcitabine+SBRT } \\
\text { to } 8 \mathrm{~Gy} \times 3 \text { fractions }\end{array}$ \\
\hline
\end{tabular}

SBRT: Stereotactic body radiation therapy

acute and late toxici-ties and can be used in patients who have had prior radiotherapy.[3]

\section{Conclusion}

Stereotactic body radiotherapy treatment is well tolerated with low toxicity rates and effective means of oligo-metastasis control. It could represent an available treatment option for oligo-metastatic patients not amenable to surgery, even when patients had been pretreated with chemotherapy.

Peer-review: Externally peer-reviewed.

Conflict of Interest: None declared.

\section{References}

1. Baliga S, Mehta K, Goldberg G, Kalnicki S. Stereotactic Body Radiation Therapy in Oligo-Metastatic Recurrent Ovarian Cancer. Int J Radiat OncolBiol Phys 2013;87(2):S425.

2. Benedict SH, Yenice KM, Followill D, Galvin JM, Hinson W, Kavanagh B, et al. Stereotactic body radiation therapy: the report of AAPM Task Group 101. Med Phys 2010;37(8):4078-101.

3. Fields EC, McGuire WP, Lin L, Temkin SM. Radiation Treatment in Women with Ovarian Cancer Past, Present, and Future. Front Oncol 2017;7:177.

4. Alongi F, Arcangeli S, Filippi AR, Ricardi U, Scorsetti M. Review and uses of stereotactic body radiation therapy for oligometastases. Oncologist 2012;17(8):11007.

5. Kunos CA, Sherertz TM, Mislmani M, Ellis RJ, Lo SS, Waggoner SE, et al. Phase I trial of carboplatin and gemcitabine chemotherapy and stereotactic ablative radiosurgery for the palliative treatment of persistent or recurrent gynecologic cancer. Front Oncol 2015;5:126.

6. Correa RJ, Salama, JK, Milano MT, Palma DA. 2016: Stereotactic Body Radiotherapy for Oligometastasis: Opportunities for Biology to Guide Clinical Management. Cancer Journal 2016;22(4):247-56.

7. Mesko S, Sandler K, Cohen J, Konecny G, Steinberg M, Kamrava M. Clinical outcomes for stereotactic ablative radiotherapy in oligometastatic and oligoprogressive gynecological malignancies. Int J Gynecol Cancer 2017;27(2):403-8. 